\title{
Tourette Syndrome: Gene Expression as a Tool to Discover Drug Targets
}

\author{
Isaac H. Liao and Frank R. Sharp \\ Medical Investigation of Neurodevelopmental Disorders (M.I.N.D.) Institute and Department of Neurology, University of \\ California at Davis, Sacramento, California 95817
}

\begin{abstract}
Summary: Gene expression microarrays are a high-throughput, cost-effective method for measuring the expression of all genes in a sample. By comparing the expression patterns of healthy controls to diseased subjects, the genetic regulatory pathways underlying and affected by the disease can be elucidated. Furthermore,
\end{abstract}

dysregulated genes are possible candidates for pharmaceutical therapy. Here, we consider the possibility of applying this approach to Tourette syndrome. We also review current theories of Tourette syndrome etiology and pharmacology. Key Words: Tourette syndrome, gene expression, microarrays, pharmacology.

\section{INTRODUCTION}

Tourette syndrome (TS) is a neurodevelopmental disorder defined by the Diagnostic and Statistical Manual of Mental Disorders, Fourth Edition, Text Revision

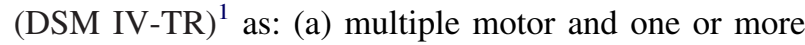
vocal tics; (b) tics that occur daily or intermittently but must be present for at least one year; and (c) onset before age 18. The severity and frequency of tics wax and wane, tend to peak in adolescence, and often improve by the end of adolescence or early adulthood. ${ }^{2-4}$

Despite decades of research, the cause of TS remains unknown. ${ }^{5}$ Many studies have shown that it is familial. ${ }^{6-10}$ However, a twin study found only a $53 \%$ concordance rate in monozygotic twins and an $8 \%$ concordance rate in dizygotic twins, showing that TS is not entirely genetically determined. ${ }^{11}$ As such, multi-factorial models have proposed that TS is a complex genetic disease resulting from a combination of an unknown number of genetic predisposition(s) and environmental trigger(s). ${ }^{8}$ Several genes have been associated with TS, including SLITRK1, CNTNAP2 and COMT. ${ }^{12-14}$ SLITRK1 and CNTNAP2 are both integral membrane proteins; SLITRK1 regulates neurite outgrowth, ${ }^{15}$ and CNTNAP2 colocalizes with potassium channels in myelinated axons. ${ }^{16}$ COMT is involved in catecholamine metabolism. Interestingly, CNTNAP2 has been associated with au-

Address correspondence and reprint requests to: Isaac Liao, Ph.D., 2805 50th Street, Sacramento, CA 95817. E-mail: ihliao@ ucdavis.edu. tism. ${ }^{17-19}$ However, associations between these genes and TS have been restricted to particular populations and subsequent studies have failed to replicate them. ${ }^{20-27}$ Genes that are associated with TS in a substantial population have yet to be identified. ${ }^{28}$

As for environmental factors, there is evidence for an association between TS and group A $\beta$-hemolytic streptococcal (GABHS) infections. A study of 41 children with TS and 39 controls found a significant increase in serum antibodies against human putamen following streptococcal infection in TS compared to controls. ${ }^{29}$ The idea that streptococcal infection triggers an autoimmune reaction, leading to the manifestation of tic disorders and OCD, is known as pediatric autoimmune neuropsychiatric disorders associated with streptococcal infections (PANDAS).$^{30}$ However, this is still controversial in TS. ${ }^{31}$ Finally, the observation that tics are exacerbated during increased psychosocial stress has led to the proposal that TS arises, in part, from increased reactivity of the hypothalamic-pituitary-adrenal (HPA) axis. ${ }^{32}$

TS is often comorbid with obsessive-compulsive disorder (OCD) and attention deficit hyperactivity disorder (ADHD). ${ }^{33}$ There may also be a connection between tic disorders and autism spectrum disorders (ASD); a clinical survey of 105 children and adolescents with ASD found that 22 percent of them also exhibited motor tics or $\mathrm{TS}^{34}$ However, the mechanisms linking TS to other neurodevelopmental disorders remain unknown.

Anatomical and functional studies have implicated the basal ganglia in TS. A MRI study found significantly 
smaller caudate nucleus volumes in TS compared to controls. ${ }^{35}$ In addition, a fMRI study found changes in basal ganglia and thalamic activity during periods of tic suppression. ${ }^{36}$ Another fMRI study found that tic severity was correlated with enhanced activation of several regions in the direct pathway through the basal ganglia in unmedicated subjects performing a cognitive control task. ${ }^{37}$ However, the causes of basal ganglia pathology in tics and TS remain unknown.

\section{PHARMACOLOGY}

TS is often treated with medications that reduce or alleviate tics. The majority of these medications fall into two categories: atypical antipsychotics and $\alpha$-2-adrenergic agonists. ${ }^{38}$

Atypical antipsychotics include risperidone, clozapine, olanzapine aripiprazole, quetiapine, and ziprasidone. These medications act in part by blocking D2 dopamine receptors in the dopaminergic nigrostriatal pathway of the basal ganglia. Based on current understanding of basal ganglia connectivity, this would increase the activity of the indirect pathway through the basal ganglia, inhibiting unwanted movements. ${ }^{37}$ However, because atypical antipsychotics also act on D4 dopamine receptors and 5-HT2A serotonin receptors, their exact mechanism of action is a source of debate.

The $\alpha$-2-adrenergic agonists, such as clonidine and guanfacine, are also used to treat tics, especially in cases of comorbid ADHD. Their effectiveness is similar to that of atypical antipsychotics. ${ }^{39}$ Although the mechanism by which $\alpha$-2a-adrenergic agonists relieve tics is unclear, stimulation of $\alpha$-2a-adrenergic receptors (ADRA2A) inhibits epinephrine release. ${ }^{40}$ The role of norepinephrine in TS circuits is unclear, though NE levels in cerebrospinal fluid (CSF) have been found to be $55 \%$ higher in un-medicated TS subjects compared to normal controls. ${ }^{41}$

The prevailing theory of TS etiology postulates an imbalance between the direct (excitatory) and indirect (inhibitory) pathways through the basal ganglia, wherein the direct pathway is overactive, the indirect pathway is less active, or both. Dopamine-releasing neurons in the substantia nigra pars compacta $(\mathrm{SNc})$ excite striatal neurons with D1 dopamine receptors in the direct pathway, but inhibit striatal neurons with D2 dopamine receptors in the indirect pathway. Thus, activation of $\mathrm{D} 2$ dopamine receptors in the striatum should lead to tics due to increased inhibition of the indirect pathway, while blockage of D2 dopamine receptors in the striatum should alleviate tics by disinhibiting the indirect pathway. As such, typical and atypical antipsychotics relieve tics by specifically blocking D2 dopamine receptors. This has been shown to increase D2 dopamine receptor mRNA. ${ }^{42}$

\section{GENE EXPRESSION MICROARRAYS}

Gene expression microarrays allow investigators to measure the amount of messenger RNA for all genes present in a sample. Microarrays consist of a substrate such as glass, with tens of thousands of spots. Bound to each spot are copies of a short nucleotide sequence (for Affymetrix GeneChip microarrays, 25 base pairs long). These probe sequences, which are different for each spot, are chosen to be identical to certain segments of mRNA. Groups of probes, all recognizing different parts of the same mRNA, are called probesets.

After the total mRNA is isolated from a tissue sample, it is reverse-transcribed to complementary DNA (cDNA). This cDNA is amplified using polymerase chain reaction (PCR) and fluorescently labeled. The labeled cDNA is then washed over the surface of a microarray, and binds to probes via complementary hybridization. The fluorescence of each spot gives an indication of how much cDNA is bound to it, and hence, how much mRNA of the corresponding sequence was present in the original sample. The fluorescence of each spot is quantified, and the resulting data is stored for analysis. Data from probes of each probeset are summarized to give probeset-level data. Probeset-level data roughly corresponds to genelevel expression data, although there are multiple probesets for some genes. Heatmaps are often used to visualize gene expression microarray data (FIG. 1).

Microarrays enable high-throughput, cost-effective measurement of gene expression. ${ }^{43}$ By examining gene expression patterns in relation to disease state, disease severity, or disease progression over time, differences in gene expression levels relevant to the disease can be identified. Such studies can provide insight into the generegulatory mechanisms of a disease, and also identify targets for pharmaceutical therapy. ${ }^{44}$ Novel techniques and resources are enabling improved analyses of gene expression data in the context of neuroscience. ${ }^{45}$

\section{GENE EXPRESSION IN TOURETTE SYNDROME}

We have measured peripheral blood gene expression to investigate TS. ${ }^{46,47}$ Assuming that TS is caused by both genetic and environmental factors, gene expression may be an ideal level of investigation, because transcriptional regulation represents the interaction of genes and the environment.

Ideally, we would like to examine gene expression in brain tissue, but peripheral blood provides a much less invasive alternative. Changes in gene expression in the brain can be seen in peripheral blood through several mechanisms. Numerous biological pathways active in the central nervous system are also active to a measurable degree in white blood cells (e.g., those related to 


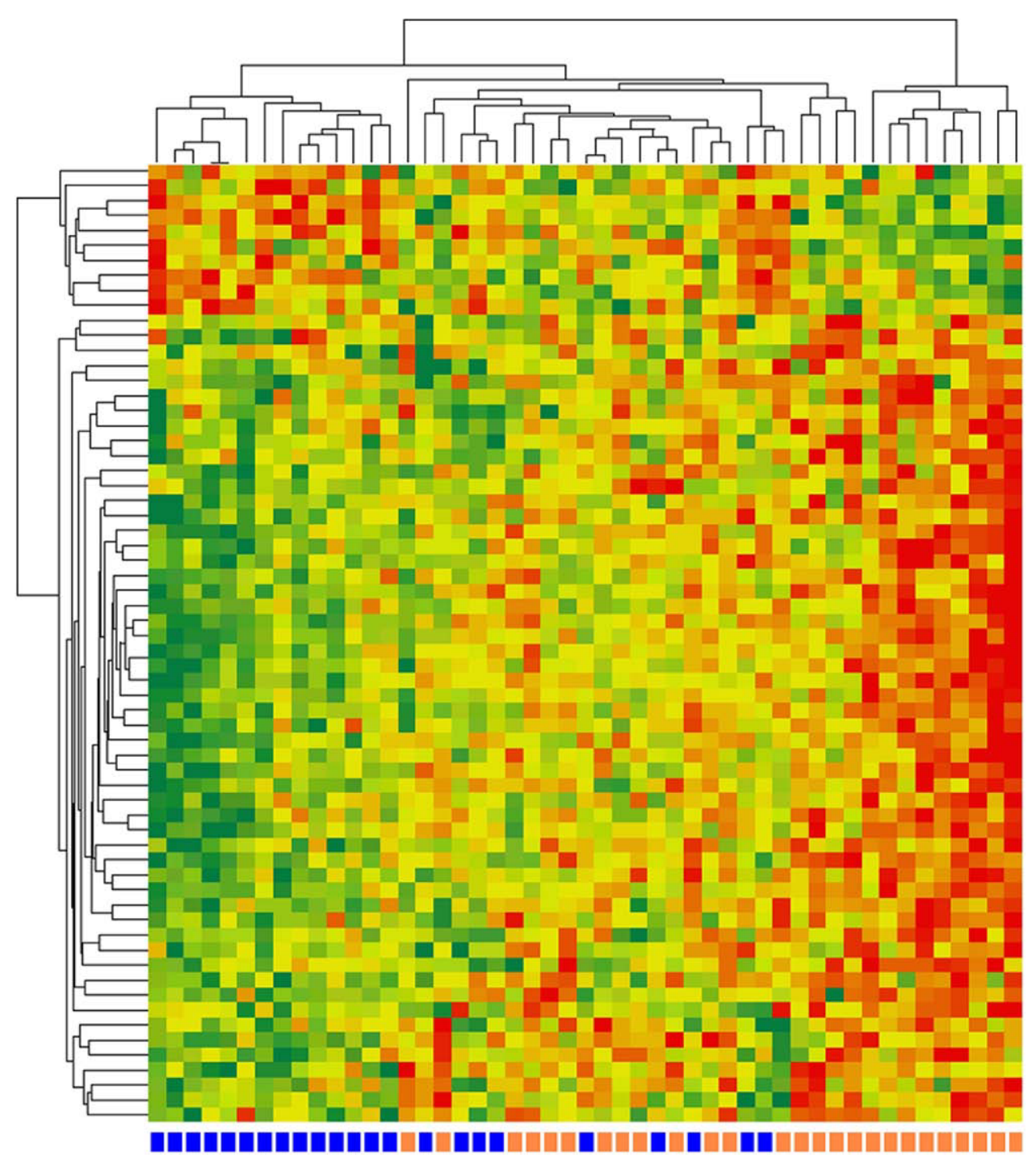

FIG 1. Gene expression microarray heat map example. Heat maps are used to visualize gene expression data. This heat map shows the standardized expression levels of 68 genes in the peripheral blood of Tourette syndrome (TS) and control subjects. Subjects are arranged in columns; genes are arranged in rows. Blue = control subjects; green = lower gene expression; orange = TS subjects; red = higher gene expression.

dopamine neurotransmission). Peripheral blood routinely interacts with the central nervous system across the blood-brain barrier. ${ }^{48-51}$ Finally, streptococcal infection and subsequent autoimmune responses may be involved in the pathophysiology of some TS individuals. ${ }^{52}$ Quantification of gene expression in white blood cells may provide further evidence to support or discredit this claim. Indeed, a previous study by our group found that natural killer cell genes were overexpressed in the peripheral blood of TS subjects. ${ }^{47}$

More recently, we examined blood gene expression in TS relative to medication status (submitted for publication). Because dopaminergic and adrenergic medications are effective in tic retreatment, we restricted our investigation to genes related to catecholamines or neurotransmission. Online databases are helpful in describing gene function, and we used two of them: 1) the Kyoto Encyclopedia of Genes and Genomes, ${ }^{53}$ and 2) the Gene Ontology ${ }^{54}$ database. We computed correlations between gene expression and tic severity for each gene. Some genes were only correlated with tic severity in TS patients who were not taking medications, and not in those who were taking medications. It is possible that the expression of these genes was affected by the patients' medications. In addition, several genes correlated with tic severity regardless of medication status. These genes could be related to tic manifestation, and their expression may not be affected by current medications. Thus, they may represent novel targets for pharmaceutical therapy in the reduction of tic severity. Finally, one dopaminerelated gene was correlated with tic severity in patients taking medications, but not in patients who were not taking 
medications. This pattern of expression is consistent with the fact that most of the medications in this study were dopamine antagonists, which are known to affect gene expression. $^{42}$

\section{CHALLENGES AND FUTURE DIRECTIONS}

There are several challenges to interpreting microarray data. The large size of the dataset is susceptible to false positives. Affymetrix Human Genome U133 Plus 2.0 microarrays consist of approximately 55,000 probesets, corresponding to approximately 35,000 genes. Thus, there are 55,000 data points per sample, and a significance level of $p<0.0001$ would still be expected to yield a number of false positives by chance. Also related to the size of the dataset is the issue of multiple comparisons. When individual data is compared between samples, or averaged data is compared between groups, the probability of detecting at least one significant gene is greater because of the large number of comparisons being made. To account for this, the Holm-Bonferroni correction $^{55}$ can be used to adjust $p$ values, making them more stringent based on the number of comparisons. However, many believe that the Bonferroni correction is too conservative for microarray data and prefer to use the Benjamini-Hochberg false discovery rate correction. ${ }^{56}$ Alternatively, investigators may restrict their search to certain genes, which are chosen a priori. This method effectively reduces the size of the dataset and the number of comparisons to a more manageable number.

Another issue that arises in microarray data analysis is that of the batch effect. A variety of factors, including the time and place of microarray processing, can introduce variance into the results. Several studies attribute at least part of this effect to ambient ozone levels during microarray hybridization, washing, and scanning. ${ }^{57-59}$ To correct for the batch effect, microarray experiments must be carefully designed, such that each microarray contains interleaved samples from each experimental condition. For example, if performing a case-control study, half of the samples on each microarray should be controls, half should be from the treatment group, and the samples should be interleaved. This ensures that the batch effect is not confounded with the factors of interest, and can later be accounted for using an analysis of covariance. ${ }^{60}$

Exon arrays are a refinement of gene expression quantification technology. Genomic DNA consists of coding regions called exons, and noncoding regions called introns. Messenger RNA transcribed from DNA consists of several exons spliced together. However, the particular exons included in an mRNA can vary among transcripts of the same gene. This phenomenon is known as alternative splicing, and different transcripts from the same gene are known as splice variants. ${ }^{61}$ Instead of measuring mRNA at the gene level, exon arrays consist of probes for every known exon. ${ }^{62}$ Thus, they are sensitive to alternatively spliced isoforms of genes. In addition, due to the greater number of probes (6.5 million for Affymetrix exon arrays), each gene can be interrogated at many sites along its length, promising greater accuracy. It will be interesting to see whether the use of exon arrays will improve the quality of gene expression data, and whether analysis of alternative splicing will provide further insights into disease mechanisms and novel targets for pharmacotherapy.

\section{REFERENCES}

1. Diagnostic and statistical manual of mental disorders (DSM-IVTR), 4th ed. Arlington, VA: American Psychiatric Assoc, 2007.

2. Swain JE, Scahill L, Lombroso PJ, King RA, Leckman JF. Tourette syndrome and tic disorders: a decade of progress. J Am Acad Child Adolesc Psychiatry 2007;46:947-968.

3. Müller N. Tourette's syndrome: clinical features, pathophysiology, and therapeutic approaches. Dialogues Clin Neurosci 2007;9:161171.

4. Singer HS. Tourette's syndrome: from behaviour to biology. Lancet Neurol 2005;4:149-159.

5. Kremer HPH. What's wrong with Tourette syndrome? Parkinsonism Relat Disord 2007;13(Suppl 3):S385-S386.

6. Pauls DL, Raymond CL, Stevenson JM, Leckman JF. A family study of Gilles de la Tourette syndrome. Am J Hum Genet 1991; 48:154-163.

7. Kano Y, Ohta M, Nagai Y, Pauls DL, Leckman JF. A family study of Tourette syndrome in Japan. Am J Med Genet 2001;105:414421.

8. Walkup JT, LaBuda MC, Singer HS, et al. Family study and segregation analysis of Tourette syndrome: evidence for a mixed model of inheritance. Am J Hum Genet 1996;59:684-693.

9. Hebebrand J, Klug B, Fimmers R, et al. Rates for tic disorders and obsessive compulsive symptomatology in families of children and adolescents with Gilles de la Tourette syndrome. J Psychiatr Res 1997;31:519-530.

10. Eapen V, Pauls DL, Robertson MM. Evidence for autosomal dominant transmission in Tourette's syndrome. United Kingdom cohort study. Br J Psychiatry 1993;162:593-596.

11. Price RA, Kidd KK, Cohen DJ, Pauls DL, Leckman JF. A twin study of Tourette syndrome. Arch Gen Psychiatry 1985;42:815820.

12. Miranda DM, Wigg K, Kabia EM, et al. Association of SLITRK1 to Gilles de la Tourette syndrome. Am J Med Genet B Neuropsychiatr Genet 2009;150B:483-486.

13. Barr CL, Wigg KG, Sandor P. Catechol-O-methyltransferase and Gilles de la Tourette syndrome. Mol Psychiatry 1999;4:492-495.

14. Verkerk AJMH, Mathews CA, Joosse M, et al. CNTNAP2 is disrupted in a family with Gilles de la Tourette syndrome and obsessive compulsive disorder. Genomics 2003;82:1-9.

15. Kajiwara Y, Buxbaum JD, Grice DE. SLITRK1 binds 14-3-3 and regulates neurite outgrowth in a phosphorylation-dependent manner. Biol Psychiatry 2009;66:918-925.

16. Poliak S, Gollan L, Martinez R, et al. Caspr2, a new member of the neurexin superfamily, is localized at the juxtaparanodes of myelinated axons and associates with K+ channels. Neuron 1999;24: $1037-1047$

17. Alarcón M, Abrahams BS, Stone JL, et al. Linkage, association, and gene-expression analyses identify CNTNAP2 as an autismsusceptibility gene. Am J Hum Genet 2008;82:150-159.

18. Arking DE, Cutler DJ, Brune CW, et al. A common genetic variant in the neurexin superfamily member CNTNAP2 increases familial risk of autism. Am J Hum Genet 2008;82:160-164.

19. Poot M, Beyer V, Schwaab I, et al. Disruption of CNTNAP2 and additional structural genome changes in a boy with speech delay and autism spectrum disorder. Neurogenetics 2010;11:81-89.

20. Barr CL, Wigg KG, Sandor P. Catechol-O-methyltransferase and Gilles de la Tourette syndrome. Mol Psychiatry 1999;4:492-495. 
21. Deng H, Le WD, Xie WJ, Jankovic J. Examination of the SLITRK1 gene in Caucasian patients with Tourette syndrome. Acta Neurol Scand 2006;114:400-402.

22. Fabbrini G, Pasquini M, Aurilia C, et al. A large Italian family with Gilles de la Tourette syndrome: clinical study and analysis of the SLITRK1 gene. Mov Disord 2007;22:2229-2234.

23. Cavallini MC, Di Bella D, Catalano M, Bellodi L. An association study between 5-HTTLPR polymorphism, COMT polymorphism, and Tourette's syndrome. Psychiatry Res 2000;97:93-100.

24. Belloso JM, Bache I, Guitart M, et al. Disruption of the CNTNAP2 gene in a $t(7 ; 15)$ translocation family without symptoms of Gilles de la Tourette syndrome. Eur J Hum Genet 2007;15:711-713.

25. Scharf JM, Moorjani P, Fagerness J, et al. Lack of association between SLITRK1var321 and Tourette syndrome in a large family-based sample. Neurology 2008;70:1495-1496.

26. Giller EL, Young JG, Breakefield XO, et al. Monoamine oxidase and catechol-O-methyltransferase activities in cultured fibroblasts and blood cells from children with autism and the Gilles de la Tourette syndrome. Psychiatry Res 1980;2:187-197.

27. Keen-Kim D, Mathews CA, Reus VI, et al. Overrepresentation of rare variants in a specific ethnic group may confuse interpretation of association analyses. Hum Mol Genet 2006;15:3324-3328.

28. Pauls D. An update on the genetics of Gilles de la Tourette syndrome. J Psychosom Res 2003;55:7-12.

29. Singer HS, Giuliano JD, Hansen BH, et al. Antibodies against human putamen in children with Tourette syndrome. Neurology 1998;50:1618-1624.

30. Swedo SE, Leonard HL, Garvey M, et al. Pediatric autoimmune neuropsychiatric disorders associated with streptococcal infections: clinical description of the first 50 cases. Am J Psychiatry 1998;155:264-271.

31. Singer HS, Hong JJ, Yoon DY, Williams PN. Serum autoantibodies do not differentiate PANDAS and Tourette syndrome from controls. Neurology 2005;65:1701-1707.

32. Corbett BA, Mendoza SP, Baym CL, Bunge SA, Levine S. Examining cortisol rhythmicity and responsivity to stress in children with Tourette syndrome. Psychoneuroendocrinology 2008;33:810-820.

33. Bloch MH, Leckman JF. Clinical course of Tourette syndrome. J Psychosom Res 2009;67:497-501.

34. Canitano R, Vivanti G. Tics and Tourette syndrome in autism spectrum disorders. Autism 2007;11:19-28.

35. Peterson BS, Thomas P, Kane MJ, et al. Basal ganglia volumes in patients with gilles de la Tourette syndrome. Arch Gen Psychiatry 2003;60:415-424.

36. Peterson BS, Skudlarski P, Anderson AW, et al. A functional magnetic resonance imaging study of tic suppression in Tourette syndrome. Arch Gen Psychiatry 1998;55:326-333.

37. Baym CL, Corbett BA, Wright SB, Bunge SA. Neural correlates of tic severity and cognitive control in children with Tourette syndrome. Brain 2008;131:165-179.

38. Srour M, Lespérance P, Richer F, Chouinard S. Psychopharmacology of tic disorders. J Can Acad Child Adolesc Psychiatry 2008; $17: 150-159$.

39. Gaffney GR, Perry PJ, Lund BC, et al. Risperidone versus clonidine in the treatment of children and adolescents with Tourette's syndrome. J Am Acad Child Adolesc Psychiatry 2002; 41:330-336.

40. Rang H. In: Pharmacology, 5th ed. Edinburgh: Churchill Livingstone, 2003:129.

41. Leckman JF, Goodman WK, Anderson GM, et al. Cerebrospinal fluid biogenic amines in obsessive compulsive disorder, Tourette's syndrome, and healthy controls. Neuropsychopharmacology 1995; 12:73-86.

42. Sands S, Dickerson D, Morris S, Chronwall B. Dopamine D2 receptor stimulation alters G-protein expression in rat pituitary intermediate lobe melanotropes. Endocrine 1997;6:325-333.

43. Schena M, Heller RA, Theriault TP, et al. Microarrays: biotechnology's discovery platform for functional genomics. Trends Biotechnol 1998;16:301-306.

44. Debouck C, Goodfellow PN. DNA microarrays in drug discovery and development. Nat Genet 1999;21(1 Suppl):48-50.

45. Geschwind DH, Konopka G. Neuroscience in the era of functional genomics and systems biology. Nature 2009;461:908-915.

46. Lit L, Enstrom A, Sharp FR, Gilbert DL. Age-related gene expression in Tourette syndrome. J Psychiatr Res 2009;43:319-330.

47. Lit L, Gilbert DL, Walker W, Sharp FR. A subgroup of Tourette's patients overexpress specific natural killer cell genes in blood: a preliminary report. Am J Med Genet B Neuropsychiatr Genet 2007;144B:958-963.

48. de Haas AH, van Weering HRJ, de Jong EK, Boddeke HWGM, Biber KPH. Neuronal chemokines: versatile messengers in central nervous system cell interaction. Mol Neurobiol 2007;36:137-151.

49. Volterra A, Meldolesi J. Astrocytes, from brain glue to communication elements: the revolution continues. Nat Rev Neurosci 2005; 6:626-640.

50. Baggiolini M. Chemokines and leukocyte traffic. Nature 1998;392: $565-568$.

51. Ransohoff RM, Kivisakk P, Kidd G. Three or more routes for leukocyte migration into the central nervous system. Nat Rev Immunol 2003;3:569-581.

52. Martino D, Dale RC, Gilbert DL, Giovannoni G, Leckman JF. Immunopathogenic mechanisms in Tourette syndrome: A critical review. Mov Disord 2009;24:1267-1279.

53. Ogata H, Goto S, Sato K, et al. KEGG: Kyoto encyclopedia of genes and genomes. Nucleic Acids Res 1999;27:29-34.

54. Blake JA, Harris MA. The Gene Ontology (GO) project: structured vocabularies for molecular biology and their application to genome and expression analysis. Curr Protoc Bioinformatics 2002 Nov; Chapter 7:Unit 7.2.

55. Holm S. A Simple Sequentially Rejective Multiple Test Procedure. Scand Stat Theory Appl. 1979;6:65-70.

56. Benjamini Y, Hochberg Y. Controlling the false discovery rate: a practical and powerful approach to multiple testing. J R Stat Soc Series B Stat 1995;57:289-300.

57. Fare TL, Coffey EM, Dai H, et al. Effects of atmospheric ozone on microarray data quality. Anal Chem 2003;75:4672-4675.

58. Branham WS, Melvin CD, Han T, et al. Elimination of laboratory ozone leads to a dramatic improvement in the reproducibility of microarray gene expression measurements. BMC Biotechnol 2007; $7: 8$.

59. Byerly S, Sundin K, Raja R, et al. Effects of ozone exposure during microarray posthybridization washes and scanning. J Mol Diagn 2009;11:590-597.

60. Ayroles JF, Gibson G. Analysis of variance of microarray data. Meth Enzymol 2006;411:214-233.

61. Keren H, Lev-Maor G, Ast G. Alternative splicing and evolution: diversification, exon definition and function. Nat Rev Genet 2010; 11:345-355.

62. Okoniewski MJ, Miller CJ. Comprehensive analysis of affymetrix exon arrays using bioconductor. PLoS Comput Biol 2008;4:e6. 